\title{
A Visual Language for Protein Design
}

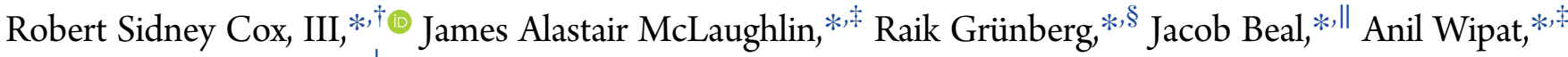 \\ and Herbert M. Sauro*, ${ }^{*}$ \\ ${ }^{\dagger}$ Material Science Institute, University of Oregon, Eugene, Oregon 97403, United States \\ ${ }^{\ddagger}$ School of Computing Science, Newcastle University, Newcastle upon Tyne NE1 7RU, U.K. \\ ${ }^{\S}$ Computational Bioscience Research Center, King Abdullah University for Science and Technology, Thuwal 23955, Saudi Arabia \\ "Raytheon BBN Technologies, Cambridge, Massachusetts 02138, United States \\ ${ }^{\perp}$ Department of Bioengineering, University of Washington, Seattle, Washington 98105, United States
}

\section{Supporting Information}

\begin{abstract}
As protein engineering becomes more sophisticated, practitioners increasingly need to share diagrams for communicating protein designs. To this end, we present a draft visual language, Protein Language, that describes the high-level architecture of an engineered protein with easy-todraw glyphs, intended to be compatible with other biological diagram languages such as SBOL Visual and SBGN. Protein Language consists of glyphs for representing important features (e.g., globular domains, recognition and localization sequences, sites of covalent modification, cleavage and catalysis), rules for composing these glyphs to represent complex architectures, and rules constraining the scaling and styling of diagrams. To support Protein Language we have implemented an extensible web-based software diagram tool, Protein Designer, that uses Protein Language in a "drag and drop" interface for visualization and computer-aided-design of engineered proteins, as well as conversion of annotated protein sequences to Protein Language diagrams and figure export. Protein Designer can be accessed at http://biocad.ncl.ac.uk/protein-designer/.
\end{abstract}

KEYWORDS: synthetic biology, visualization, Synthetic Biology Open Language, genetic circuits, protein engineering

$\mathrm{P}$ rotein engineering is one of the oldest disciplines of molecular biotechnology, with a rich history of engineering by mutation and fusion of genes coding for functional protein sequences. As more sophisticated and model-driven methods have become available, practitioners need to communicate increasingly complex designs. In other disciplines, such as electrical engineering ${ }^{1,2}$ or architecture and mechanical engineering, ${ }^{3,4}$ standard visual symbols and diagram languages allow engineers to more easily comprehend designs, avoid mistakes, build software tools, etc. No standard visual language has been proposed, however, for the depiction of design features within individual engineered proteins. We address this by presenting a draft visual language for protein design, Protein Language.

Protein Language is specifically intended to aid protein design and not to describe all existing knowledge of protein biology. This approach is in keeping with other visual languages in engineering disciplines: for example, electronics diagrams do not aim to capture the full range of electromagnetic phenomena and architectural diagrams do not aim to describe the full physics of built structures. Accordingly, we have created glyphs focused on a subset of design elements intended to cover many of the most common changes that protein engineers make to manipulate protein function, expression and production. Protein Language has been simultaneously developed with the aim of compatibility with other standards in biological engineering, including the Systems Biology Graphical Notation $(\mathrm{SBGN})^{5}$ and the Synthetic Biology Open Language Visual (SBOLv). ${ }^{6,7}$ Thus, Protein Language makes use of design standards from other fields to produce a distinct and clear visual style, while remaining largely compatible with related efforts.

Protein Language provides users with a wide range of expressive capabilities, which can improve communication of protein designs with rapidly drawn, easy to interpret, highquality technical diagrams. To support use and adoption of Protein Language, we have also implemented a web-based software tool, Protein Designer, that provides an accessible interface for using these symbols to construct diagrams. We plan for Protein Language and its symbols to be adjusted and further refined through the experience of practitioners and an open community standardization process.

\section{RESULTS}

Protein Language and Glyph Set. At present, there are 12 glyphs defined for Protein Language: four region glyphs and

Special Issue: IWBDA 2016

Received: October 18, 2016

Published: February 7, 2017 


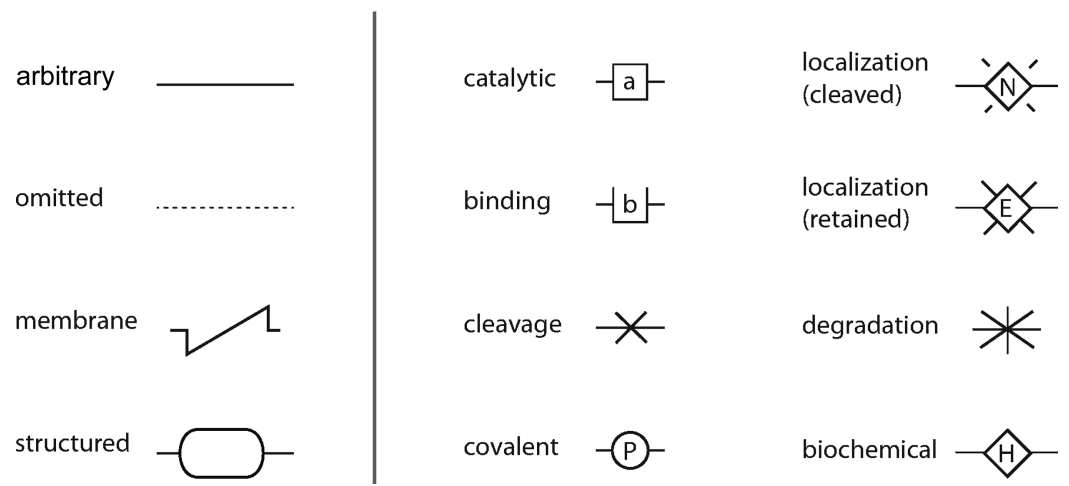

Figure 1. Glyphs defined for Protein Language: four protein region glyphs and eight protein site glyphs.

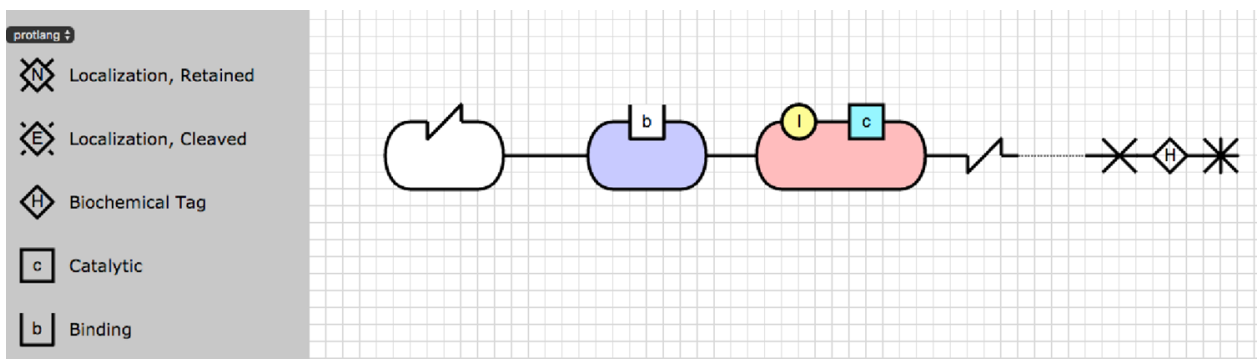

Figure 2. Protein Designer is a web-based software tool for construction of Protein Language diagrams using scalable vector graphics.

eight site glyphs. These glyphs have been chosen to be compatible with existing literature where possible, plus a number of novel symbols intended to be clear, easy to draw, and easy to distinguish. All 12 glyphs are shown in Figure 1 and described in detail in Supporting Appendix A. These glyphs are intended to serve as general categories for design rather than formal ontological definitions. For example, a region containing several transmembrane domains could be represented as several membrane glyphs, as a single structured region glyph, or omitted altogether with the omitted protein region glyph, depending on what a practitioner wishes to communicate regarding that sequence. Together, the 12 glyphs can generate a wide range of conceivable protein designs.

A Protein Language diagram is built around a straight line, a common literature representation of an amino acid chain. Other significant features of the protein are then represented by "region" glyphs ordered along this backbone and "site" glyphs that are ordered along a region. The backbone line represents an arbitrary protein region, with unspecified structural properties. Unstructured and linker regions are normally shown as a backbone line. A rectangle with rounded edges describes a structured protein region, such as a protein domain, consistent with typical conventions from the literature on protein domains (e.g., refs 8,9 ). The width of the rectangle may be scaled to indicate relative region size. Membrane regions are shown with a zigzag line, inspired by several literature illustrations. ${ }^{10,11}$ This membrane glyph can be used on either the backbone or the structured protein region glyph. We also include a dotted line to describe a region that is present in the protein but omitted from the diagram. These four region glyph types describe variably sized protein regions, are consistent with previous literature descriptions, and allow the user to highlight the basic structure of globular domains, disordered regions, and membrane regions.

Smaller significant features of a protein's structure and function are represented by eight site glyphs, representing features from one to 30 amino acids in length. The catalytic glyph represents an enzyme active site or binding pocket. The binding glyph is used to represent protein binding to various ligands including protein, DNA, and small molecules. The cleavage glyph covers proteolytic sites, and the similar degradation glyph includes recognition sites for processive protein degradation machinery and systems such as ubiquitination. Protein modifications by covalent attachment of small molecules are represented by the covalent glyph, covering posttranslational modifications such as phosphorylation-a focus of intense research in the proteomic literature (e.g., refs 10, 12). Two localization glyphs allow for the description of C-terminal, $\mathrm{N}$-terminal, or internal sequences for protein transport, allowing protein designs to specify cellular location. Finally, the biochemical tag glyph includes sites for protein purification, crystallization, and other chemical handles. The eight site glyphs thus describe enzyme active sites and locations where a protein is post-translationally modified, cleaved, degraded, binded, transported, or biochemically manipulated.

Protein Designer. Protein Designer is a web-based software tool for creating and manipulating Protein Language diagrams, available at http://biocad.ncl.ac.uk/protein-designer/ . A screenshot is shown in Figure 2. (Note: At present, Protein Designer requires the Google Chrome or Chromium desktop browser.) The user can create a protein backbone (arbitrary region) by right clicking on the blank canvas. An unlimited number of resizable backbone lines are supported. The sidebar allows the user to select a glyph from the glyph set, which can then be placed on the canvas or attached to a protein backbone. The structured protein region glyph, in turn, has its own backbone attachment points for adding site glyphs to the top or bottom. Once completed, designs can be exported, using the button located in the top right, into Scalable Vector Graphic $\left(\mathrm{SVG}^{13}\right)$ images. The SVG can also be converted to PDF by the browser's print dialogue, and either form imported into compatible illustration or presentation software. Protein 
Designer's simple interface allows fast layout of designs using Protein Language.

Protein Designer uses a modular system of drawing rules to render SVG. New glyphs can be defined as geometrical rules using the SVG commands for path drawing: moveto, lineto, and closepath (ref 13 Section 8.3). This allows users the option of contributing new glyphs to the language as SVG geometry definitions, which can be incorporated into the Protein Designer code. The architecture of Protein Designer allows new glyphs and sets of glyphs to be added easily, which we hope will facilitate the development of a standard visual protein language.

Example A: Protease Sensor. Figure 3 shows a Protein Language diagram representing a protease-based sensor. ${ }^{14}$ This

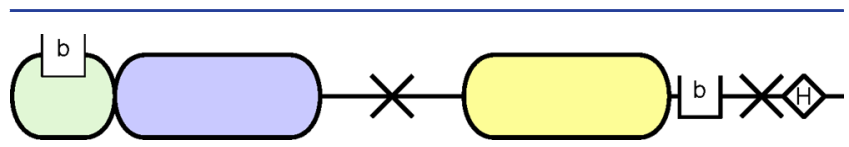

Figure 3. Diagram example: protease FRET sensor combining fluorescent reporter domains (mTurquoise and mCitrine, shown in blue and yellow, respectively) connected with a disordered region containing a protein cleavage site for caspase 3 protease, which is active at the onset of apoptosis. An N-terminal WW domain (green) forms an intramolecular noncovalent interaction with a Proline-rich peptide motif located close to the protein's C-terminus. These two binding sites are shown by the noncovalent binding glyph ("b"). A second cleavage site at the C-terminus allows for the cleavage of a biochemical purification tag (hexahistidine, " $\mathrm{H}$ ").

protein device consists of regions encoding two colors of fluorescent proteins with a disordered region between them. Inside the disordered region is a protein cleavage site. This sensor exhibits fluorescent resonance energy transfer (FRET) between the two fluorescent protein domains, which is abolished when the protein is cleaved. The FRET signal is enhanced through a noncovalent binding: an intramolecular "helper interaction."

Example B: Light-Inducible Protein Membrane Localization. Figure 4 shows a Protein Language diagram
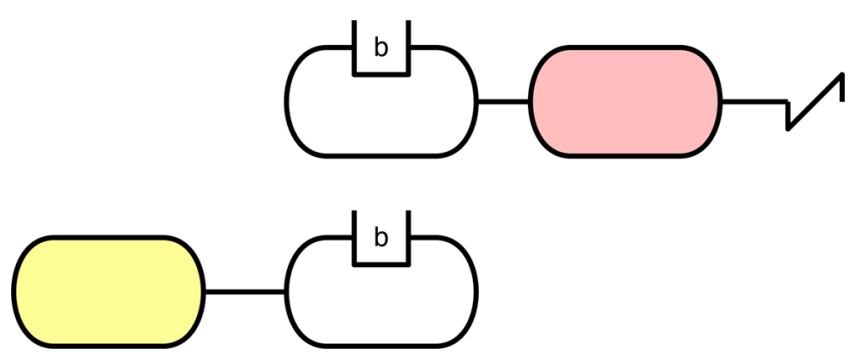

Figure 4. Diagram example: the light-inducible PIF domain is used to create a reporter system for programmed localization of proteins to the plasma membrane. The protein binding domain ("b") is modulated by light reversibly when exposed to red $(650 \mathrm{~nm})$ or infrared $(750 \mathrm{~nm})$ light. Each protein backbone also contains a distinct fluorescent reporter protein, mCitrine (yellow) and mCherry (red).

representing light-inducible protein membrane localization. ${ }^{15}$ This system consists of two separate protein backbones that can be brought together via a light-induced conformational change that reversibly controls protein binding. Two fluorescent reporter domains are used to image the localization of each protein to the cell plasma membrane, where one of the proteins is anchored by a membrane region. The system can be used as a general, reversible system for regulated recruitment in eukaryotes.

Example C: Inducible Artificial Transcription Factor. Figure 5 shows a Protein Language diagram representing an inducible

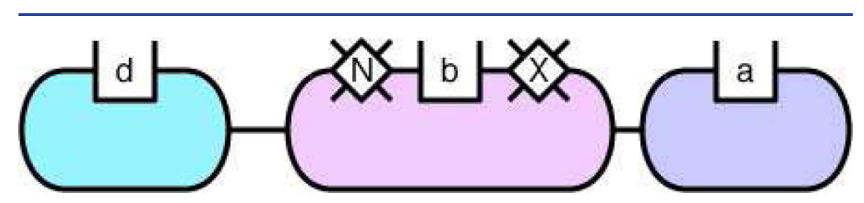

Figure 5. Diagram example: an estrogen receptor region is used to add an inducible response to an artificial transcription factor. This design brings together three protein regions: the $\mathrm{N}$-terminus encodes a DNA binding domain ("d", a zinc finger DNA recognition region binding to a specific 9 base-pair DNA sequence); the middle region contains the estrogen receptor, which controls nuclear localization of the entire protein with an inducible response to the hormone beta-estradiol; the C-terminus encodes the activation domain VP16 ("a"), which recruits polymerase to activate a eukaryotic promoter. The nuclear localization is modulated by a retained nuclear localization signal " $N$ " and a retained nuclear export signal " $\mathrm{X}$ " where " $\mathrm{N}$ " is blocked when bound to Hsp90 and unblocked when bound to estrogen.

artificial transcription factor. ${ }^{16}$ The estrogen receptor region is used to add inducible response to an artificial transcription factor. This design incorporates three structured protein regions: a DNA binding domain, the estrogen receptor, and a eukaryotic activation domain. Each domain's function is described by site glyphs for binding and localization.

\section{DISCUSSION}

Visual depictions have always been an important tool in the design of biological systems. We have presented the first diagram language for constructing visualizations specifically for purposes of protein engineering. Rather than focusing on protein structure, as in protein ribbon diagrams, ${ }^{17}$ Protein Language operates at a higher level of functional abstraction. This abstraction to the modular aspects of protein design reflects the increasing sophistication of protein engineering models, allowing the communication between practitioners to focus on the primary functional characteristics of a design and leaving the specific details of its realization to be examined as necessary. As protein engineering capabilities improve, we expect that abstract design diagrams will become increasingly important. Concurrently, as protein engineering capabilities improve, we expect that Protein Language will expand to cover a large range of routinely engineered features.

The immediate next steps we envision for this effort, however, focus on refinement of Protein Language and its integration with existing standards and communities. In particular, we aim to integrate Protein Language with the Systems Biology Graphical Notation $(\mathrm{SBGN})^{5}$ and Synthetic Biology Open Language Visual (SBOLv) ${ }^{6,7}$ standards, both of which are free and open standards supported by diverse international communities and part of the COmputational Modeling in Blology NEtwork (COMBINE) federated standards collection. Together, SBOLv and SBGN enable canonical depictions of functional pathways, structural features of DNA, and biochemical interactions, but presently neither has a means of depicting the substructure of a protein-a complementary capability provided by Protein Language. Moreover, efforts already underway in both of these communities will facilitate integration with Protein Language: SBGN is being enhanced to support diagram elements that show the substructure of 
chemical species using other visual languages, and SBOLv is being enhanced to support the standardized depiction of nonnucleic-acid components. As there are a number of minor differences in how Protein Language is currently formulated and the rules of these standards, integration will involve a number of refinements and adjustments. Given the positive reception that Protein Language has received in initial community discussions, however, we have confidence that it will ultimately form the basis for a broadly accepted, community-supported open standard that helps to effectively integrate engineered proteins into the design of biological systems.

\section{ASSOCIATED CONTENT}

\section{S Supporting Information}

The Supporting Information is available free of charge on the ACS Publications website at DOI: 10.1021/acssynbio.6b00286.

Glyph images in SVG format (ZIP)

Glyph images in PDF format (ZIP)

Supporting Appendices (PDF)

\section{AUTHOR INFORMATION}

\section{Corresponding Authors}

*E-mail: sidney@dna.caltech.edu.

*E-mail: j.a.mclaughlin@newcastle.ac.uk.

*E-mail: raik.gruenberg@gmail.com.

*E-mail: jakebeal@ieee.org.

*E-mail: anil.wipat@ncl.ac.uk.

*E-mail: uw.hsauro@gmail.com.

ORCID

Robert Sidney Cox III: 0000-0002-2009-6236

Notes

The authors declare no competing financial interest.

\section{ACKNOWLEDGMENTS}

The authors thank Steven Schkolne for consultation on glyph design and Matthew Pocock and Christopher Voigt for helpful discussions. J.A.M. is supported by FUJIFILM DioSynth Technologies. A.W. is supported by the Engineering and Physical Sciences Research Council grant EP/J02175X/1 and EP/N031962/1. R.S.C. is supported in part by US DoD grant FA5209-16-P-0041 and National Science Foundation grant DBI-1355909. J.S.B. is supported in part by the National Science Foundation Expeditions in Computing Program Award \#1522074 as part of the Living Computing Project. This document does not contain technology or technical data controlled under either the U.S. International Traffic in Arms Regulations or the U.S. Export Administration Regulations.

\section{REFERENCES}

(1) IEEE (1991) IEEE Graphic Symbols for Logic Functions (Includes IEEE Std 91A-1991 Supplement, and IEEE Std 91-1984). IEEE Std. 91a-1991.

(2) IEEE (1993) IEEE Standard American National Standard Canadian Standard Graphic Symbols for Electrical and Electronics Diagrams (Including Reference Designation Letters). IEEE Std. 3151975 (Reaffirmed 1993).

(3) Schley, M., Buday, R., Sanders, K., and Smith, D. (1997) AIA $C A D$ Layer Guidelines; The American Institute of Architects Press: Washington, D.C.

(4) British Standards Institution (2007) Collaborative Production of Architectural, Engineering and Construction Information. BS 1192:2007.
(5) le Novere, N., Hucka, M., Mi, H., Moodie, S., Schreiber, F., Sorokin, A., Demir, E., Wegner, K., Aladjem, M. I., and Wimalaratne, S. M. (2009) The systems biology graphical notation. Nat. Biotechnol. 27, $735-741$.

(6) Quinn, J. Y., et al. (2015) SBOL Visual: A Graphical Language for Genetic Designs. PLoS Biol. 13, e1002310.

(7) Quinn, J., Beal, J., Bhatia, S., Cai, P., Chen, J., Clancy, K., Hillson, N., Galdzicki, M., Maheshwari, A., Pocock, M., Rodriguez, C., Stan, G.B., and Endy, D. (2013) Synthetic Biology Open Language Visual (SBOL Visual), version 1.0.0

(8) Chen, C., Nott, T. J., Jin, J., and Pawson, T. (2011) Deciphering arginine methylation: Tudor tells the tale. Nat. Rev. Mol. Cell Biol. 12, 629-642.

(9) Lai, A., Sato, P. M., and Peisajovich, S. G. (2015) Evolution of synthetic signaling scaffolds by recombination of modular protein domains. ACS Synth. Biol. 4, 714-722.

(10) Choudhary, C., and Mann, M. (2010) Decoding signalling networks by mass spectrometry-based proteomics. Nat. Rev. Mol. Cell Biol. 11, 427-439.

(11) Lim, W. A. (2010) Designing customized cell signalling circuits. Nat. Rev. Mol. Cell Biol. 11, 393-403.

(12) Whitaker, W. R., Davis, S. A., Arkin, A. P., and Dueber, J. E. (2012) Engineering robust control of two-component system phosphotransfer using modular scaffolds. Proc. Natl. Acad. Sci. U. S. A. $109,18090-18095$

(13) Ferraiolo, J., Jun, F., and Jackson, D. (2000) Scalable Vector Graphics (SVG) 1.0 Specification; iuniverse.

(14) Grünberg, R., Burnier, J. V., Ferrar, T., Beltran-Sastre, V., Stricher, F., van der Sloot, A. M., Garcia-Olivas, R., Mallabiabarrena, A., Sanjuan, X., Zimmermann, T., and Serrano, L. (2013) Engineering of weak helper interactions for high-efficiency FRET probes. Nat. Methods 10, 1021-1027.

(15) Levskaya, A., Weiner, O. D., Lim, W. A., and Voigt, C. A. (2009) Spatiotemporal control of cell signalling using a light-switchable protein interaction. Nature 461, 997-1001.

(16) McIsaac, R. S., Oakes, B. L., Wang, X., Dummit, K. A., Botstein, D., and Noyes, M. B. (2013) Synthetic gene expression perturbation systems with rapid, tunable, single-gene specificity in yeast. Nucleic Acids Res. 41, e57.

(17) Richardson, J. S. (1985) Schematic drawings of protein structures. Methods Enzymol. 115, 359-380. 